\title{
Improved Resource Allocation for TV White Space Network Based on Modified Firefly Algorithm
}

\author{
Kennedy K. Ronoh ${ }^{1}$, and George Kamucha ${ }^{2}$, Thomas O. Olwal ${ }^{3}$ and \\ Tonny K Omwansa ${ }^{1}$
}

${ }^{1}$ School of Computing and Informatics, University of Nairobi, Nairobi, Kenya

${ }^{2}$ Department of Electrical and Information Engineering, University of Nairobi, Nairobi, Kenya

${ }^{3}$ Department of Electrical Engineering, Tshwane University of Technology, Pretoria, South Africa

There is continued increased demand for dynamic spectrum access of TV White Spaces (TVWS) due to growing need for wireless broadband. Some of the use cases such as cellular (2G/3G/4G/5G) access to TVWS may have a high density of users that want to make use of TVWS. When there is a high density of secondary users $(S U s)$ in a TVWS network, there is possibility of high interference among $S U$ s that exceeds the desired threshold and also harmful interference to primary users (PUs). Optimization of resource allocation (power and spectrum allocation) is therefore necessary so as to protect PUs against harmful interference and to reduce the level of interference among $S U$ s. Existing resource allocation optimization algorithms for a TVWS network ignore adjacent channel interference, interference among $S U$ s or apply greedy algorithms which result in sub-optimal resource allocation. In this paper we propose an improved resource allocation algorithm based on continuous-binary firefly algorithm. Simulation is done using Matlab. Simulation results show that the proposed algorithm improves the $S U$ sum throughput and $S U$ signal to interference noise (SINR) ratio in the secondary network.

ACM CCS (2012) Classification: Networks $\rightarrow$ Network components $\rightarrow$ Wireless access points, base stations and infrastructure $\rightarrow$ Cognitive radios

Keywords: dymamic spectrum access, cognitive radio, spectrum allocation, power control

\section{Introduction}

Spectrum occupancy assessments done in USA, Spain, Singapore, New Zeland and Germany [1] and UK [2], indicate that a large portion of spectrum assigned to primary users ( $P U \mathrm{~s})$ is underutilized. Spectrum is considered a scarce re- source. More and more devices want a pie of the spectrum and yet the useful spectrum is limited. Dynamic Spectrum Access (DSA), through the use of Cognitive Radio (CR) techniques is currently being embraced as a solution to spectrum underutilization and spectrum scarcity. This is because DSA, together with $\mathrm{CR}$, provides an efficient way for spectrum management and spectrum sharing. DSA allows the existence of both primary and secondary users in a non-interfering basis. With DSA, spectrum allocated for exclusive use to a Primary User $(P U)$ but being not used by the $P U$ (incumbent), or any other idle frequency bands (such as guard bands) can be shared by different secondary users $(S U$ s) as long as the interference to the incumbent by the $S U$ s to the $P U$ is kept at an acceptable level [3]. The spectrum band which has attracted a lot interest in the DSA community is the TVWS. TVWS is the spectrum band not being utilized efficiently by TV transmitters in the Ultra High Frequency (UHF) band. The main reason for this increased interest is the good propagation characteristics of the sub-1 GHz spectrum.

Regulatory authorities worldwide have mandated the use of geo-location database (GLDB) for protection of $P U$ s. GLDB is used by a $S U$ or white space device (WSD) to find the set of frequency channels that can be used on a secondary basis in a given area and at any given time [4]. GLDB is populated through the use of a propagation model. The database contains estimated power levels of incumbents ( $P U \mathrm{~s})$ for any point in a particular region of interest. The WSD, 
which has a cognitive radio system (CRS), queries a central database. The WSD provides the database with parameters such as its location, device type and antenna height. The GLDB will then use this information along with the parameters of all surrounding TV transmitters such as antenna height, transmit power and frequency of operation in order to come up with the list of available TVWS channels that can be used by the WSD on secondary basis without causing harmful interference to the PUs. The GLDB will also give the WSD limits on the transmit power and also the time period in which each channel can be used.

It is expected that there will be continued demand for dynamic spectrum access (DSA). There is increased demand for DSA to TVWS from internet of things (IoT) [5], machine to machine communications, vehicle to vehicle (V2V) communications [6], [7], cellular networks (3G , 4G, 5G) [8], [9], [10]. This increased demand for DSA will result in secondary networks with a high density of users. The problem of interference will arise in a TVWS network with a high density of users. Some $S U$ s also may not be admitted into the secondary network due to interference constraints at $P U$ s and SUs. TVWS can be used as long as the interference to the $P U$ does not fall below a certain threshold. This threshold is commonly referred to as protection ratio or desired to undesired $(\mathrm{D} / \mathrm{U})$ ratio.

In a network where there is a high number of devices seeking access to a secondary network allocation of two resources, power and spectrum, has to be optimized to ensure that as many SUs as possible access the secondary network while ensuring that interference constraints for $P U \mathrm{~s}$ and QoS requirements for $S U$ s are met. In this paper, resource allocation refers to joint allocation of power and spectrum to $S U$ s.

Existing algorithms allocate spectrum and power in a one by one, greedy manner as SUs make request to the GLDB. This will result in sub-optimal resource allocation. Existing algorithms also ignore adjacent channel interference (ACI). When there is a high density of devices in a network, ACI cannot be ignored even if the $S U$ s are using low power since aggregate interference from multiple $S U$ s using the same channel is as harmful as co-channel interference [11],
[12], [13]. SUs also have minimum QoS/interference constraints measured by SINR. There is, therefore, a need for an algorithm that can be used to optimize resource allocation for all existing users in the secondary network so as to maximize the number of users (both $S U$ s and $P U \mathrm{~s})$ in the network that meet the required QoS/interference constraints.

Firefly algorithm (FA) will be used for optimization of resource allocation. FA is chosen because it has been found out that it performs better than other metaheuristic algorithms such as particle swarm optimization and genetic algorithm [14]. The aim of this paper, therefore, is to design an improved algorithm for resource allocation based on modified FA for a TVWS network. We propose a hybrid continuous-binary FA since the optimization involves both continuous values (power allocation) and binary values (spectrum allocation). A TVWS network with a base station is considered.

The contribution of this paper is the design of an improved algorithm based on modified FA for joint spectrum allocation and transmit power control in a GLDB based wireless TVWS network where devices communicate via a base station. To the best of our knowledge, continuous-binary FA has not been used for joint spectrum allocation and power control in a TVWS network that makes use of a GLDB. Simulation results show that the use of the algorithm results in improvement sum throughput and SU SINR.

The rest of the paper is organized as follows. In Section 2, related work is discussed. System model has been presented in Section 3. Simulation set up and simulation parameters have been presented in Section 4. Performance analysis of the proposed algorithm is discussed in Section 5. The paper is concluded in Section 6.

\section{Related Work}

A resource allocation method has been proposed in IEEE 802.11af [15]. In an IEEE 802.11af network, a device sends a channel availability query (CAQ) to registered location secure server (RLSS). RLSS operates as a GLDB. Once a CAQ is received by the RLSS, it will respond with a white space map (WSM). The WSM contains the list of available channels and their respective effective isotropic radiated 
power (EIRP). IEEE 802.11af allows for both closed loop power control and open loop power control. With open loop power limitation the WSD has rigid power limitation similar to those provided by FCC regulations [3], [16] whereby fixed power values are assigned to $S U$ s. In closed loop power control, the WSD has more flexible power limits that depend on location, time of use and the channel. The technique proposed in IEEE 802.11af is not designed to optimize resource allocation as it seeks to ensure that specific users that request a channel are allocated one with an associated power level. In our proposed algorithm, resource allocation is done for all users that already exist in the network.

IEEE 802.22 makes use of a spectrum manager (SM) to allocate spectrum [17]. IEEE 802.22 allows the use of both GLDB and spectrum sensing for incumbent protection. The SM makes use of spectrum sensing function and GLDB to find out the channels available for secondary use and their respective effective isotropic radiated power (EIRP) limits. Just like IEEE 802.11af, the technique proposed in IEEE 802.22 is not designed to optimize resource allocation as it seeks to ensure that specific users that request channel are allocated one with an associated power level. Power and spectrum allocation is done in an arbitrary manner with no use of an objective function. It will not be applicable in a high density network where there is a need to optimize resource allocation so as to admit as many users as possible into the network.

GLDB based spectrum allocation with power control, co-channel interference and adjacent channel interference considerations has been proposed by Xue et al. [18]. Co-existence (mutual interference) among $S U$ s is also considered. Channel allocation and power control is then done in such a manner that the TV receiver and SUs SINR constraints are met. A greedy algorithm is used for power control and spectrum allocation. Each $S U$ is allocated a channel and a power level when it makes a channel request to the GLDB. The major disadvantage of the proposed algorithm is that, being a greedy algorithm, it may get trapped in a local optimum. Being trapped in local optimum will result in sub-optimal resource allocation.

GLDB based spectrum allocation with power control and admission control for TVWS multiple device-to-device links has been proposed by Xue et al. [19]. Only co-channel interference has been considered. Spectrum allocation is done in greedy manner using a game theory algorithm called spatial adaptive play (SAP). The algorithm has the following disadvantages. Firstly, being a greedy algorithm, it may get trapped in a local optimum. Being trapped in local optimum will result in sub-optimal resource allocation. Secondly, the algorithm ignores ACI.

\section{System Model}

We proposed a power control algorithm in [14]. We extend the model and algorithm to incorporate both power and spectrum allocation. A network illustrated by Figure 1 is considered. In the figure there is a single TV receiver placed at the edge of the protection region. Among all the TV receivers in the protection region, a $\mathrm{TV}$ receiver at this location is the one which is most vulnerable to interference since it is very close to the secondary network and receives the weakest signal from the TV tower. GLDB regulations require that the $\mathrm{D} / \mathrm{U}$ ratio or protection ratio be measured at the edge of protection region [20]. Aggregate interference at the TV receiver, both co-channel and adjacent channels should not make the protection ratio fall below the required protection ratio threshold.

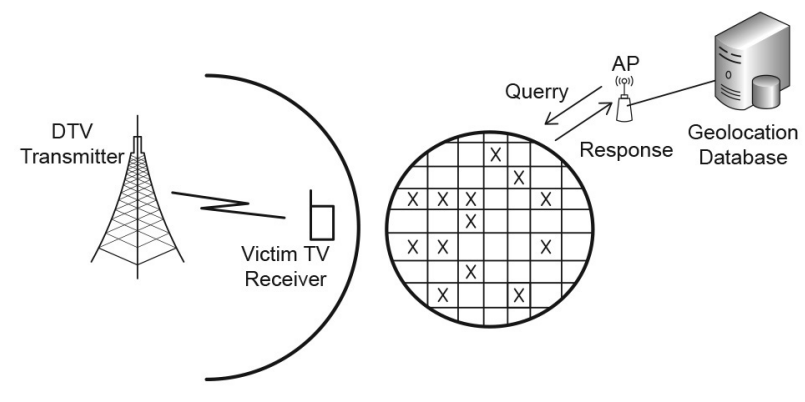

Figure 1. Interference scenario.

Let the number $S U$ s be $N$ and the number of channels be $M$. Let the potential channel allocation matrix be represented as $A=\left\{a_{n, m} \| a_{n, m}\right.$ $\in\{0,1\}\} . A$ is of dimension $N \times M \cdot a_{n, m}=1$ if channel $m$ assigned to user $n . a_{n, m}=0$ if channel $m$ is not assigned to user $n$. Let the potential 
power allocation vector be $P=\left\{P_{m}{ }^{1}, P_{m}{ }^{2}, \ldots\right.$, $\left.P_{m}{ }^{n}, \ldots, P_{m}{ }^{N}\right\}$ where $P_{m}{ }^{n}$ is the power of SU $n$ on channel $m$. Assuming that the TV receiver operates using channel $c_{T V}$ at frequency $\left(f_{c_{T V}}\right)$, the interference by a single $S U n$ to the TV receiver can be written as [21],[18]:

$$
I_{T V, n}=\mu\left(C_{T V}, C_{n}\right) P_{n}^{C_{n}} G_{n}^{S U \rightarrow P U} G_{S U} G_{P U I}
$$

where $P_{n}^{c_{n}}$ is the transmit power of $S U n$ is operating on channel $c_{n}, G_{n}^{S U \rightarrow P U}$ is the path loss from $S U n$ to the victim TV receiver, $G_{S U}$ is the antenna gain of $S U$ and $G_{P U}$ is the antenna gain of the $P U$ (TV receiver). The term $\mu\left(c_{T V}, c_{n}\right)$ refers to adjacent channel interference co-efficient defined in [14], [18], [21]. If adjacent channel interference is modeled as equivalent co-channel interference, the total interference to the $P U$ can then be expressed as:

$$
I_{T V}=\sum_{n=1}^{N} I_{T V, n} .
$$

Equation (3) represents the SINR at the TV receiver. In equation (3), the term $\omega_{o}$ is the minimum required SINR at the TV receiver, $P_{T V}$ is the received power from the TV transmitter at the TV receiver and $\delta_{n}^{2}$ is noise power.

$$
\frac{P_{T V}}{I_{T V}+\delta_{n}^{2}} \geq \omega_{o} \text {. }
$$

Every single $S U$ will receive interference from other $S U$ s. The interference at $S U n$ using channel $c_{n}$ from all other $S U$ s in the network using channel $c_{j}$ is denoted as:

$$
I_{S U_{n}}=\sum_{\substack{j=1, j \neq n}}^{N} I_{n, j}=\sum_{\substack{j=1, j \neq n}}^{N} P_{j}^{c_{j}} G_{j}^{n} G_{S U},
$$

where $I_{n, j}$ is the interference caused by $S U j$ to $S U n, G_{j}^{h}$ is the distance based path loss from $S U j$ to $S U n$. SINR at each $S U$ can then be written as:

$$
\rho_{n} \frac{P^{B S} G_{S U} G_{B S}}{I_{S U_{n}}+\delta_{n}^{2}} \geq \rho_{o},
$$

where $P^{B S}$ is the transmit power of the access point (base station) and $G^{B S}$ is the antenna gain of the base station. The term $\rho_{o}$ is the minimum required SINR at $S U$ s. The downlink throughput $r_{n, m}$ of $S U$ transmitter $n$ on channel $m$ can be expressed as:

$$
r_{n, m}=\frac{1}{2} a_{n, m} b_{m} \log _{2}\left(1+\rho_{n}\right) .
$$

The sum throughput of all SUs will then be given by:

$$
U=\sum_{n=1}^{N} \sum_{m=1}^{M} r_{n, m}
$$

In order to minimize interference to the $P U$ and among $S U$ s, there is a need for joint spectrum and a power allocation algorithm. The optimization goal is to find a power vector $P^{*}$ and channel allocation matrix $A^{*}$ that maximizes sum downlink throughput while ensuring that interference constraints at the $P U$ and all $S U$ s are met. The power of each $S U$ is adjusted between the range $\left[P_{\min }, P_{\max }\right]$. Optimal power vector and spectrum allocation matrix can be found by solving optimization Problem 1 below.

Problem 1.

$$
P^{*}, A^{*}=\arg \max (U),
$$

subject to:

$$
\begin{gathered}
C_{1}: \omega>\omega_{o}, \\
C_{2}: \rho_{n}>\rho_{o}, n=1,2, \ldots, N, \\
C_{3}: p_{\min } \leq p_{n} \leq p_{\max }, \\
C_{4}: a_{n, m} \in\{0,1\}, \\
C_{5}: a_{n, m}=1, c_{n}=m, \\
C_{6}: a_{n, m}=0, c_{n} \neq m,
\end{gathered}
$$

where $\omega$ is SINR at the TV receiver. Constraints $C_{5}$ and $C_{6}$ imply that one channel $\left(c_{n}=m\right)$ only in $S U_{n}$ channel allocation row will have the value 1 , the rest will be 0 .

Pseudocode for the FA is presented in [14], [22], [23]. Flash flies produce short and rhythmic light to attract a female partner and potential prey. Each firefly's attractiveness is proportional to the light intensity and decreases as distance increases [22], [23]. Variation of attractiveness with distance is given by: 


$$
\beta=\beta_{o} e^{-\gamma r^{2}} .
$$

For any two flashing fireflies, the less bright one will move towards the brighter one according to following equation:

$$
x_{i}^{t+1}=x_{i}^{t}+\beta_{o} e^{-\gamma r_{i j}^{2}}\left(x_{j}^{t}-x_{i}^{t}\right)+\alpha_{t} \varepsilon_{t}^{i} .
$$

The terms $x_{i}$ and $x_{j}$ are the locations of firefly $i$ and firefly $j$, respectively. The symbol $\alpha$ is a randomization parameter and the term $\varepsilon_{t}^{i}$ is a vector of random numbers with uniform distribution. The first term $\left(\beta_{o} e^{-\gamma r_{i j}^{2}}\left(x_{j}^{t}-x_{i}^{t}\right)\right)$ represents attractiveness while the second term $\left(\alpha_{t} \varepsilon_{t}^{i}\right)$ represents randomization. Symbol $t$ is the iteration number. The distance between fireflies, $r_{i j}$, is computed as follows:

$$
r_{i j}=\sqrt{\sum_{d=1}^{D}\left(x_{d, i}-x_{d, j}\right)^{2}},
$$

where $D$ is the dimension of the solution. $D=N$ for the problem under consideration.

Since optimization problem is about joint optimization of power and spectrum allocation, each firefly, $x_{i}$, will be made up of a power vector $\left(x_{P, i}\right)$ and a spectrum allocation matrix $\left(x_{C, i}\right)$. Equation (17) applies when the values being considered are continuous. It will, therefore, apply only to the power vector. It will not apply to the spectrum allocation matrix because the values in the matrix are binary ( 0 or 1$)$. The following equation will be used for computing the distance $\left(r_{i j, C}\right)$ between two channel allocation matrices:

$$
r_{i j, C}=\sum_{d=1}^{D} \sum_{m=1}^{M} x_{C m, d, i} \otimes x_{C m, d, j},
$$

where $x_{C m, d, i}$ and $x_{C m, d, j}$ are the channel allocation values in fireflies $i$ and $j$, respectively at position $\mathrm{m}, \mathrm{d}$ in the channel allocation matrix. The following equation will be used for computing distance between two power vectors:

$$
r_{i j, P}=\sqrt{\sum_{d=1}^{D}\left(x_{P d, i}-x_{P d, j}\right)^{2}},
$$

where $x_{P d, i}$ and $x_{P d, j}$ are the power vectors in fireflies $i$ and $j$, respectively.
New power vector and channel matrix for each firefly, $x_{i}$, will be computed according to equation (20) and (21), respectively.

$$
\begin{gathered}
x_{P d, i}^{t+1}=x_{P d, i}^{t}+\beta_{o} e^{-\gamma r_{i j, P}^{2}}\left(x_{P d, j}^{t}-x_{P d, i}^{t}\right)+\alpha_{t} \varepsilon_{t}^{i} . \\
x_{C_{m, d, i}}^{t+1}=x_{C_{m, d, i}}^{t}+\beta_{o} e^{-\gamma r_{i, C}^{2}}\left(x_{C_{m, d, j}}^{t}-x_{C_{m, d, i}}^{t}\right)+\alpha_{t} \varepsilon_{t}^{i} .
\end{gathered}
$$

Since the channel allocation matrix is made up of binary values, equation (21) will not apply for the channel allocation matrix since firefly movement results in values that are not binary.

Algorithm 1. Joint power and spectrum allocation using firefly algorithm.

Step 1:

Specify $M, N$

Set the dimension of fireflies $D$

Step 2:

Initialize the control parameters of the algorithm $\alpha, \beta, \gamma$, number of fireflies NP and maximum number of iterations $t_{\max }$.

Generate initial position of each firefly $\left(x_{i}\right)$

randomly with each firefly consisting of power vector and channel vector:

Set of power vectors in the fireflies:

$x_{P}=\left[x_{P 1}, x_{P 2}, \ldots, x_{P i}, \ldots, x_{P, N P}\right]$ and $i \in(1, \ldots, N P)$

Set of channel vectors in the fireflies:

$x_{C}=\left[x_{C 1}, x_{C 2}, \ldots, x_{C i}, \ldots, x_{C, N P}\right]$ and $i \in(1, \ldots, N P)$

Step 3:

Check firefly $x_{i}$ to see if the power values in the power vector are within range. If any values are out of range then create random values that are within range to replace them.

Randomly select a single channel for each $S U$, if there is assignment of more than one channel to a $S U$.

Step 4:

Calculate the brightness/fitness value of each firefly using equation (6) and rank the fireflies according to their fitness values. Find the current best solution.

Step 5:

For every firefly, move it to the better solution according to equation (16) through application of equations (20) for power mobility and (21), (22) and (23) for channel matrix mobility.

Step 6:

If it reaches the predefined maximum number of iterations, derive the spectrum and power allocation vector of the current best solution mentioned in step 4 and stop the progress, else go to step 3 and continue. 
In order to determine whether $x_{C_{m, d, i}}$ will be 0 or 1 , Sigmoid function is first used to change the value after the firefly mobility by the channel matrices into a probability value between $[0,1]$ :

$$
\operatorname{sig}\left(x_{C_{m, d, i}}\right)=\frac{1}{1+x_{C_{m, d, i}}} .
$$

The value for each position in the channel allocation matrix is then computed as follows:

$$
x_{C_{m, d, i}}^{t+1}= \begin{cases}1 & \text { rand }()<\operatorname{sig}(f) \\ 0 & \text { else }\end{cases}
$$

where $f=x_{C_{m, d, i}}^{t}$.

The power and channel allocation problem defined in Problem 1 is a constrained optimization problem. The most common way to deal with constraints when using evolutionary algorithms to solve optimization problems is to use an exterior penalty function [24]. Exterior penalty functions are preferred over interior penalty functions because they do not require an initial feasible solution. Penalty functions change a constrained optimization problem into an unconstrained optimization problem. This is achieved by adding to the objective function a penalty term that prescribes a high cost for violation of constraints. The objective function of optimization Problem 1 will change to:

$$
\phi=U-c_{s} \sum_{i=1}^{N} \max \left[0, g_{i}^{s}\right]^{2}-c_{p} \max \left[0, g_{i}^{p}\right]^{2},
$$

where $c_{p}$ and $c_{s}$ are co-efficients for the two penalty terms, $g_{i}^{s}=\rho_{o}-\rho_{i}$ and $g_{i}^{p}=\omega_{o}-\omega_{i}$. The optimization Problem 1 can then be re-written as that in Problem 2.

\section{Problem 2.}

$$
P^{*}, A^{*}=\arg \max \phi
$$

subject to

$$
\begin{gathered}
C_{1}: p_{\min } \leq p_{n} \leq p_{\max }, \\
C_{2}: a_{n, m} \in\{0,1\}, \\
C_{3}: a_{n, m}=1, c_{n}=m, \\
C_{4}: a_{n, m}=0, c_{n} \neq m .
\end{gathered}
$$

Problem 2 is then solved using algorithm 1.

\section{Simulation Set Up}

Parameters used in the simulation are outlined in Table 1. Simulation was done using Matlab $\mathrm{R} 2016 \mathrm{a}$. Matlab is chosen because it is rich in in-built functions. $S U \mathrm{~s}(N=1000$ and $N=500)$ are distributed over an area of $1 \mathrm{~km}^{2}$. Figure 2 shows the network diagram generated in Matlab. Initially $S U$ s are distributed across 10 channels i.e. $M=10$. Initial power assignment is also done randomly.

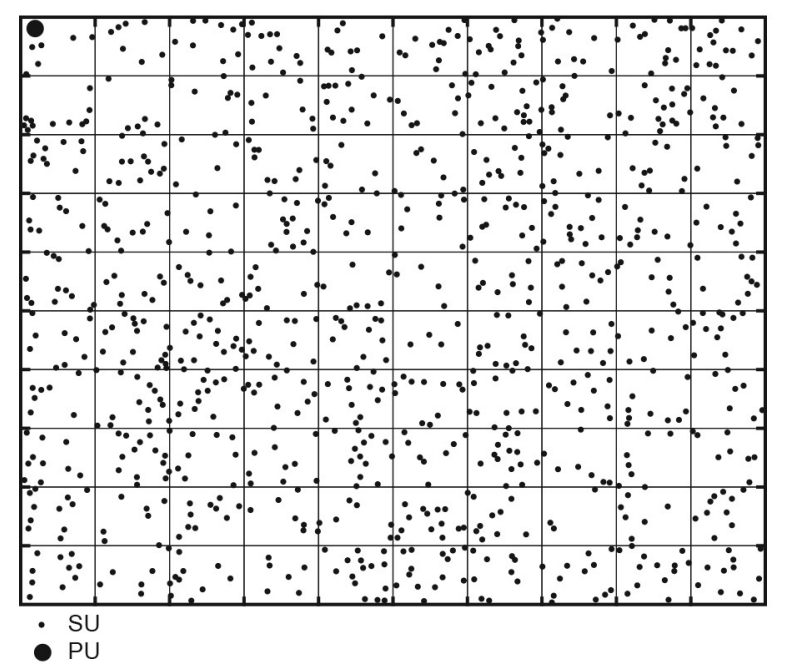

Figure 2. Network diagram.

The free space path loss model was used to model path loss:

$$
P L(d)=20 \log (d)+20 \log (f)-147.55,
$$

where $d$ is the distance in meters and $f$ is the frequency of operation. The proposed resource allocation algorithm is then used to assign power and spectrum to $S U$ s.

\section{Performance Analysis}

In order to evaluate the performance of FA, its performance is compared with those of two other joint power and spectrum allocation algorithms: SAP (spatial adaptive play, proposed in [19]) and HA (heuristic algorithm, proposed in [18]). Performance of FA algorithm is also compared with those of two other metaheuristic 
Table 1. Simulation parameters.

\begin{tabular}{|c|c|c|}
\hline Parameter & Value & Description \\
\hline$b_{m}$ & $6 \mathrm{MHz}$ & Bandwidth of TV channel \\
\hline$f_{a}$ & $650 \mathrm{MHz}$ & Centre frequency of DTV signal \\
\hline$P_{T V}$ & $-70.6 \mathrm{dBm}$ & Power of DTV signal at victim TV receiver \\
\hline$\delta_{n}^{2}$ & $-102 \mathrm{dBm}$ & Noise power \\
\hline$\omega_{o}$ & $23 \mathrm{~dB}$ & TV receiver SINR threshold \\
\hline$\rho_{o}$ & $7 \mathrm{~dB}$ & SU SINR threshold \\
\hline$P^{B S}$ & $36 \mathrm{dBm}(4 \mathrm{~W})$ & Transmit power of base station \\
\hline$p_{\max }$ & $30 \mathrm{dBm}$ & Maximum SU transmit power \\
\hline$\mu\left(c_{T V}, c_{n}\right)$ & $0,-28 \mathrm{~dB}$ & Adjacent channel interference co-efficient \\
\hline$G_{S U}$ & $10 \mathrm{~dB}$ & $S U$ antenna gain \\
\hline$G_{P U}$ & $10 \mathrm{~dB}$ & $P U$ antenna gain \\
\hline$G_{B S}$ & $10 \mathrm{~dB}$ & Access point antenna gain \\
\hline$\beta_{o}$ & 1 & FA parameter \\
\hline$\alpha$ & 30 & FA parameter \\
\hline$\gamma$ & 10 & FA parameter \\
\hline$N P$ & 20 & Number of fireflies \\
\hline$c_{s}$ & 1000 & Penalty term for $S U$ interference \\
\hline$c_{p}$ & 1000 & Penalty term for $P U$ interference \\
\hline
\end{tabular}

algorithms: genetic algorithm (GA) and particle swarm optimization (PSO). Results are generated for 10 simulation runs and then an average is computed for each performance metric under consideration.

Parameters used for FA are as follows: $\beta_{o}=1$, $\alpha=30, \gamma=10$, number of fireflies $N P=50$. Parameters used for PSO are as follows: number of particles $=50$, inertia weights: $w_{\max }=4$, $w_{\min }=2$, social parameter $c_{1}=2$ and cognitive parameter $c_{2}=2$. Parameters used for GA are as follows: number of chromosomes $=50$, mutation rate $=0.8$ and selection rate $=0.5$.

\subsection{Sum Throughput Analysis}

Table 2 shows comparison of the five algorithms in terms of sum throughput. For $M=$ 500 , the sum throughput of FA is $2.5 \%, 2.5 \%$, $190 \%$ and $10.7 \%$ higher than that of GA, PSO, HA and SAP, respectively. For $M=1000$, the sum throughput of FA is $13.9 \%, 11.25 \%, 24.8 \%$
Table 2. Comparison of sum throughput for different algorithms.

\begin{tabular}{|c||c|c|c|}
\hline $\begin{array}{c}\text { Number } \\
\text { of SUS }\end{array}$ & Algorithm & $\begin{array}{c}\text { Sum } \\
\text { thoughput } \\
\text { (Mb/s) }\end{array}$ & $\begin{array}{c}\text { Percentage } \\
\text { improvement }\end{array}$ \\
\hline \hline \multirow{4}{*}{500} & FA & 5120 & \\
\cline { 2 - 4 } & GA & 4994 & $2.5 \%$ \\
\cline { 2 - 4 } & PSO & 4994 & $2.5 \%$ \\
\cline { 2 - 4 } & HA & 1757 & $190 \%$ \\
\cline { 2 - 4 } & SAP & 4624 & $10.7 \%$ \\
\hline \multirow{3}{*}{1000} & FA & 5535 & \\
\cline { 2 - 4 } & GA & 4859 & $13.9 \%$ \\
\cline { 2 - 4 } & PSO & 4975 & $11.25 \%$ \\
\cline { 2 - 4 } & HA & 4435 & $24.8 \%$ \\
\cline { 2 - 4 } & SAP & 5236 & $5.7 \%$ \\
\hline
\end{tabular}

and $5.7 \%$ higher than that of GA, PSO, HA and SAP, respectively. These results show that FA achieves better throughput compared to all the rest of the algorithms. This is because FA re- 
sults in more optimal power and spectrum allocation that minimizes interference and hence maximizes throughput.

\subsection{SU SINR Analysis}

Table 3 shows comparison of performance of FA and the rest of algorithms under consideration in terms of percentage of SUs below the required SU SINR threshold of $\rho_{o}=7 \mathrm{~dB}$. For $\mathrm{M}=500$, number of $S U$ s below SINR threshold for GA, PSO, HA and SAP is higher by $1.2 \%$, $1.2 \%, 13.2 \%$ and $1.2 \%$ respectively. For $\mathrm{M}=$ 1000 , number of $S U$ s below SINR threshold for GA, PSO, HA and SAP is higher by $5.4 \%$, $5.3 \%, 13.2 \%$ and $5.4 \%$, respectively. Results, therefore, for both $\mathrm{M}=500$ and $\mathrm{M}=1000$ show that FA has lower percentage of $S U$ s that fall below the $7 \mathrm{~dB}$ minimum SINR threshold compared to the rest of the algorithms.

Comparison of performance of the algorithms in terms of cumulative distribution $S U$ SINR is shown Figure 3 and Figure 4. Figure 3 shows cumulative distribution of $S U$ SINR for $\mathrm{M}=$ 500. Figure 4 shows cumulative distribution of $S U$ SINR for $M=1000$. The two figures also show that FA always has a higher percentage of $S U$ s above most of the SINR values. This is because FA has a more optimal resource allocation compared to the rest of the algorithms.

Table 3. Comparison of percentage of $S U$ s below $S U$ SINR threshold for different algorithms.

\begin{tabular}{|c||c|c|c|}
\hline \multirow{2}{*}{$\begin{array}{c}\text { Number } \\
\text { of } \boldsymbol{S} \boldsymbol{U} \text { s }\end{array}$} & Algorithm & $\begin{array}{c}\text { Percentage } \\
\text { of } \boldsymbol{S} \text { Us below } \\
\text { 7dB SINR } \\
\text { threshold }\end{array}$ & $\begin{array}{c}\text { Percentage } \\
\text { improvement } \\
\text { by FA }\end{array}$ \\
\hline \hline \multirow{4}{*}{500} & FA & 27.4 & \\
\cline { 2 - 4 } & GA & 28.6 & $1.2 \%$ \\
\cline { 2 - 4 } & PSO & 28.6 & $1.2 \%$ \\
\cline { 2 - 4 } & HA & 84.8 & $57.4 \%$ \\
\hline \multirow{3}{*}{1000} & SAP & 28.6 & $1.2 \%$ \\
\cline { 2 - 4 } & FA & 52.4 & \\
\cline { 2 - 4 } & GA & 57.8 & $5.4 \%$ \\
\cline { 2 - 4 } & PSO & 57.1 & $5.3 \%$ \\
\cline { 2 - 4 } & HA & 65.6 & $13.2 \%$ \\
\hline
\end{tabular}

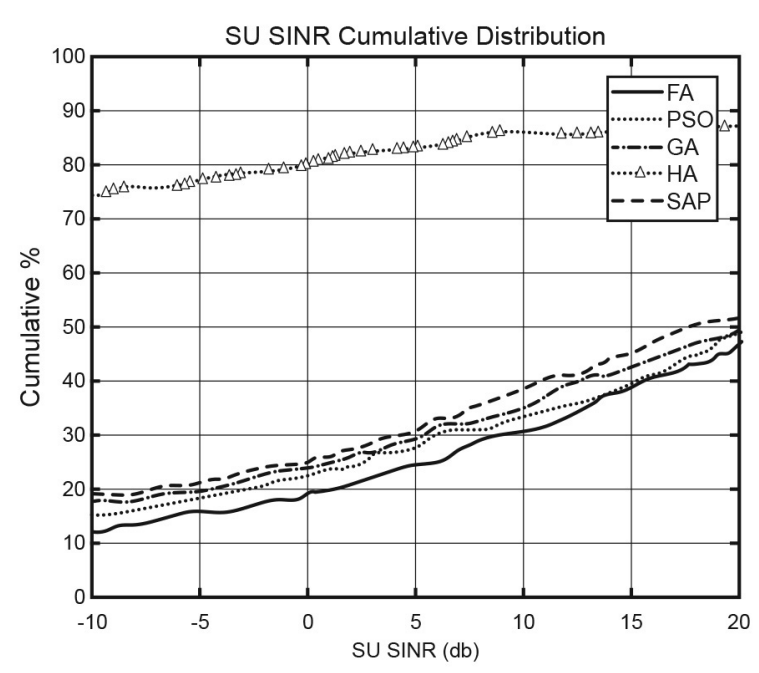

Figure 3. SU SINR distribution for $N=500$.

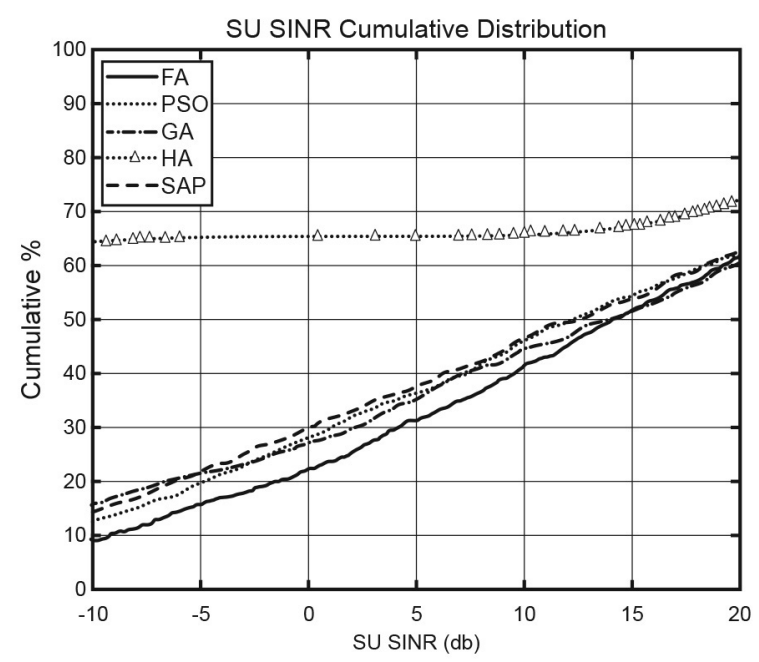

Figure 4. SU SINR distribution for $N=1000$.

\subsection{PU SINR Analysis}

Table 4 shows performance comparison of the proposed algorithm with other algorithms in terms of $P U$ SINR.

For $M=500$ and $M=1000$, the $P U$ at the edge of protection region if fully protected since $P U$ SINR is greater than the $23 \mathrm{~dB}$ minimum threshold. Results show no significant difference in performance in terms of $P U$ SINR between FA and the rest of the algorithms. This is mainly because the $P U$ penalty term is very low compared to other terms in equation (24) 
Table 4. Comparison of PU SINR for different algorithms.

\begin{tabular}{|c||c|c|c|}
\hline $\begin{array}{c}\text { Number } \\
\text { of } S U \mathbf{s}\end{array}$ & Algorithm & $\begin{array}{c}\text { PU SINR } \\
\text { (dB) }\end{array}$ & $\begin{array}{c}\text { Percentage } \\
\text { Improvement } \\
\text { by FA }\end{array}$ \\
\hline \hline \multirow{3}{*}{500} & FA & 128.43 & \\
\cline { 2 - 4 } & GA & 132.34 & $-3.91 \%$ \\
\cline { 2 - 4 } & PSO & 132.34 & $-3.91 \%$ \\
\cline { 2 - 4 } & HA & 132.63 & $-4.2 \%$ \\
\cline { 2 - 4 } & SAP & 129.34 & $-0.91 \%$ \\
\hline \multirow{3}{*}{1000} & FA & 129.96 & \\
\cline { 2 - 4 } & GA & 132.11 & $-2.15 \%$ \\
\cline { 2 - 4 } & PSO & 129.74 & $-0.22 \%$ \\
\cline { 2 - 4 } & HA & 127.83 & $-2.13 \%$ \\
\cline { 2 - 4 } & SAP & 128.86 & $+1.1 \%$ \\
\hline
\end{tabular}

\subsection{Running Time Comparison}

In order to compare the run time of algorithms, Matlab timeit() function is used. Each of the five algorithm's function handles was passed to the function. The specifications for the computer used to run the simulations are as follows: 64 bit Windows 7 operating system, 4GB RAM and $2.5 \mathrm{GHz}$ dual-core processor. The function timeit() calls a measured function several times and then returns a median of the computed running time for a measured function.

Results of running time comparison are shown in Table 5. Results show that, in terms of response time, FA is better that GA (requires $219 \%$ more time) and SAP (requires $80 \%$ more time). HA has the lowest response time (requires 68\% less time compared to FA). Although HA has lower running time, results of throughput and SU SINR show that HA results

Table 5. Comparison of running for different algorithms.

\begin{tabular}{|c||c|c|c|}
\hline Algorithm & $\begin{array}{c}\text { Run time } \\
\text { (seconds) }\end{array}$ & $\begin{array}{c}\text { Difference } \\
\text { (seconds) }\end{array}$ & $\begin{array}{c}\text { Percentage } \\
\text { difference } \\
\text { with FA }\end{array}$ \\
\hline \hline FA & 303 & & \\
\hline GA & 969 & +666 & $+68 \%$ \\
\hline PSO & 303 & 0 & 0 \\
\hline HA & 119 & -184 & $-154 \%$ \\
\hline SAP & 1518 & 1215 & $+80 \%$ \\
\hline
\end{tabular}

in very poor resource allocation. FA and PSO have no difference in terms of running time, but FA has better resource allocation since it achieves higher throughput and results in better $S U$ SINR performance compared to PSO.

\section{Conclusion}

In this paper we have proposed a continuous-binary firefly algorithm for resource allocation in a GLDB based TVWS network. Results show that the proposed algorithm results in improved sum throughput and $S U$ SINR. Compared to other algorithms, the running time of the proposed algorithm is better, except to that of PSO.

The proposed algorithm also shows that the FA and other metaheuristic algorithms, such as GA and PSO, can be applied whenever the optimization variables consist of both binary and continuous variables.

Future work will be to analyze the effect of changing FA parameters, how to choose the best channel when firefly movement result in allocation of more than one channel and testing the proposed algorithm in a real world TVWS network in order to validate its performance.

\section{References}

[1] K. Patil et al., "A Survey of Worldwide Spectrum Occupancy Measurement Campaigns for Cognitive Radio", in International Conference on Devices and Communications (ICDeCom), 2011, pp. 1-5.

http://dx.doi.org/10.1109/ICDECOM.2011.5738472

[2] M. Mehdawi et al., "Spectrum Occupancy Survey in HULL-UK for Cognitive Radio Applications: Measurement and Analysis", Int. J. Sci. Technol. Res., vol. 2, no. 4, pp. 231-236, 2013.

[3] M. Nekovee et al., "Worldwide Trends in Regulation of Secondary Access to White Spaces Using Cognitive Radio", Wirel. Commun. IEEE, vol. 19, no. 4, pp. 32-40, 2012.

http://dx.doi.org/10.1109/MWC.2012.6272421

[4] J. Heo et al., "Mobile TV White Space with Multi-Region Based Mobility Procedure", IEEE Wirel. Commun. Lett., vol. 1, no. 6, pp. 569-572, 2012.

http://dx.doi.org/10.1109/WCL.2012.081312.120314

[5] A. Aijaz and A. H. Aghvami, "Cognitive Machine-to-Machine Communications for Inter- 
net-of-Things: A Protocol Stack Perspective", IEEE Internet Things $J$., vol. 2, no. 2, pp. 103-112, 2015.

http://dx.doi.org/10.1109/JIOT.2015.2390775

[6] O. Altintas et al., "Demonstration of Vehicle to Vehicle Communications Over TV White Space", in Vehicular Technology Conference (VTC Fall), 2011, pp. 1-3.

http://dx.doi.org/10.1109/VETECF.2011.6093306

[7] S. Chen et al., "On Optimizing Vehicular Dynamic Spectrum Access Networks: Automation and Learning in Mobile Wireless Environments", presented at the Vehicular Networking Conference, 2011.

http://dx.doi.org/10.1109/VNC.2011.6117122

[8] P. Demestichas et al., "5G on the Horizon: Key Challenges for the Radio-Access Network", IEEE Veh. Technol. Mag., vol. 8, no. 3, pp. 47-53, 2013. http://dx.doi.org/10.1109/MVT.2013.2269187

[9] C. F. Silva et al., "Extension of LTE Operational Mode Over TV White Spaces", Future Netw. Mob. Summit, pp. 1-13, 2011.

[10] M. Khalil et al. "Feasibility, Architecture and Cost Considerations of Using TVWS for Rural Internet Access in 5G", in 20th Conference on Innovations in Clouds, Internet and Networks (ICIN), 2017, pp. 23-30 http://dx.doi.org/10.1109/ICIN.2017.7899245

[11] E. Obregon et al., "A Model for Aggregate Adjacent Channel Interference in TV White Space", in Vehicular Technology Conference (VTC Spring), 2011 IEEE 73rd, 2011, pp. 1-5.

http://dx.doi.org/10.1109/VETECS.2011.5956237

[12] L. Shi et al., "Controlling Aggregate Interference under Adjacent Channel Interference Constraint in TV White Space", 2012.

[13] S. Kusaladharma and C. Tellambura, "Aggregate Interference Analysis for Underlay Cognitive Radio Networks", IEEE Wirel. Commun. Lett., vol. 1, no. 6, pp. 641-644, 2012.

http://dx.doi.org/10.1109/WCL.2012.091312.120600

[14] R. Kennedy et al., "Firefly Algorithm based Power Control in Wireless TV White Space Network", in AFRICON, 2017 IEEE, 2017, pp. 155-160.

http://dx.doi.org/10.1109/AFRCON.2017.8095473

[15] A. B. Flores et al., "IEEE 802.11 af: A Standard for TV White Space Spectrum Sharing", IEEE Commun. Mag., vol. 51, no. 10, pp. 92-100, 2013. http://dx.doi.org/10.1109/MCOM.2013.6619571

[16] Technical and Operational Requirements for the possible operation of Cognitive Radio Systems in the 470-790 MHz, Eoropean Communications Commission, Cardiff, 2011.

[17] C. Cordeiro et al., "IEEE 802.22: the First Worldwide Wireless Standard based on Cognitive Radios", in First IEEE International Symposium on
New Frontiers in Dynamic Spectrum Access Networks, DySPAN 2005, 2005, pp. 328-337.

[18] Z. Xue et al., "Coexistence among Device-to-device Communications in TV White Space based on Geolocation Database", in International Workshop on High Mobility Wireless Communications (HMWC), 2014, pp. 17-22.

http://dx.doi.org/10.1109/HMWC.2014.7000206

[19] Z. Xue and L. Wang, "Geolocation Database based Resource Sharing Among Multiple Device-to-device Links in TV White Space", presented at the International Conference on Wireless Communications and Signal Processing (WCSP), 2015, pp. $1-6$.

http://dx.doi.org/10.1109/WCSP.2015.7341116

[20] D. Gurney et al., "Geo-location Database Techniques for Incumbent Protection in the TV White Space", in 3rd IEEE Symposium on New Frontiers in Dynamic Spectrum Access Networks, DySPAN 2008, 2008, pp. 1-9.

http://dx.doi.org/10.1109/DYSPAN.2008.31

[21] Z. Xue, "Geolocation Spectrum Database Assisted Coexistence of Multiple Device-to-device in TV White Space", J. Inf. Comput. Sci., vol. 12, no. 11, pp. 4443-4456, 2015.

[22] K. K. Anumandla et al., "Spectrum Allocation in Cognitive Radio Networks using Firefly Algorithm", in International Conference on Swarm, Evolutionary and Memetic Computing, 2013, pp. 366-376.

https://doi.org/10.1007/978-3-319-03753-0_33

[23] Q. Liu et al., "Spectrum Allocation Optimization for Cognitive Radio Networks Using Binary Firefly Algorithm", in Proceedings of the 2014 International Conference on Innovative Design and Manufacturing, Quebec, Canada, 2014.

http://dx.doi.org/10.1109/IDAM.2014.6912704

[24] L. A. Vardhan and A. Vasan, "Evaluation of Penalty Function Methods for Constrained Optimization using Particle Swarm Optimization", in IEEE Second International Conference on Image Information Processing (ICIIP), 2013, pp. 487-492. http://dx.doi.org/10.1109/ICIIP.2013.6707639 
Contact addresses:

Kennedy K. Ronoh

School of Computing and Informatics

University of Nairobi

Nairobi, Kenya

e-mail: ronoh.kennedy.k@ieee.org

George Kamucha

Department of Electrical and Information Engineering

University of Nairobi

Nairobi, Kenya

e-mail: gkamucha@uonbi.ac.ke

Thomas O. Olwal

Department of Electrical Engineering Tshwane University of Technology

Pretoria, South Africa

e-mail: olwalto@tut.ac.za

Tonny K. Omwansa

School of Computing and Informatics

University of Nairobi

Nairobi, Kenya

e-mail: tomwansa@uonbi.ac.ke
KenNedy K. Ronoh is currently pursuing $\mathrm{PhD}$ in Computer Science at the University of Nairobi, Kenya. He is also a Lecturer at Technical University of Kenya (Department of Computer Science and Technology), and a member of IEEE. Ronoh received his Masters in Electrical Engineering (Wireless Networks and Electronics) from Linkoping University, Sweden in 2012. His current research interests are TV white spaces, cognitive radio and wireless community networks.

George Kamucha received his PhD degree in Electrical Engineering from Kassel University, Germany in 2003. He is currently a Senior Lecturer and Chair of Department, Department of Electrical and Information Engineering, University of Nairobi, Kenya. His current research interests include communication systems and biomedical systems.

Thomas O. Olwal a senior member IEEE, received his $\mathrm{PhD}$ in Computer Science from the University of Paris-EST, France, in 2010, and the title Doctor in Technology: Electrical Engineering from Tshwane University of Technology (TUT), South Africa, in 2011. He is a registered professional engineer and currently works at TUT as an Associate Professor. His research interests include analysis and design of the spectrum, energy-efficient radio resource management, Internet of Things, advanced wireless sensor networks, SDN, Cognitive Radios, TV White spaces and Intelligent Networks. He has published over 115 technical and scientific research outputs in peer reviewed accredited journal articles, book chapters and conference papers. He also serves as a reviewer in a number of ACM/IEEE conferences and journals.

Tonny K. OMwansa is the founding Director of the C4DLab, University of Nairobi's Innovation Hub. He is also the founder and chairman of the Nairobi Innovation Week which brings numerous stakeholders shaping Kenya's Innovation Ecosystem. He lectures at the School of Computing and Informatics, University of Nairobi in Kenya and is the co-author of "Money, Real Quick: Kenya's disruptive mobile money innovation". He holds a PhD in Information Systems in which he researched the adoption of mobile financial services at the base of the pyramid in Kenya. Besides consultancy services in technology and innovation issues, he is very active in research and capacity building. His research interests are in the design, adoption and impact of innovative low-cost and appropriate technologies in developing countries. 